\title{
Chronic Blockade of Nitric Oxide Synthesis in the Rat Produces Systemic Hypertension and Glomerular Damage
}

\author{
Christine Baylis, Brij Mitruka, ${ }^{*}$ and Aihua Deng \\ Departments of Physiology and *Pathology, West Virginia University, Morgantown, West Virginia 26506
}

\begin{abstract}
Tonic basal release of nitric oxide (NO) by vascular endothelial cells controls blood pressure (BP) in the basal state. In these studies we investigated the effects of chronic inhibition of basal NO synthesis in the rat for a 2 -mo period. Significant systemic hypertension developed in chronically NO-blocked rats compared to controls. Marked renal vasoconstriction was also observed with elevations in glomerular blood pressure $\left(\boldsymbol{P}_{\mathrm{GC}}\right)$ and reductions in the glomerular capillary ultrafiltration coefficient $\left(K_{f}\right)$. Chronically NO-blocked rats also develop proteinuria and glomerular sclerotic injury compared to controls. These studies therefore describe a new model of systemic hypertension with glomerular capillary hypertension and renal disease due to chronic blockade of endogenous NO synthesis. These observations highlight the importance of the endogenous NO system in control of normal vascular tone and suggest that hypertensive states may result from relative $\mathrm{NO}$ deficiency. (J. Clin. Invest. 1992. 90:278-281.) Key words: nitric oxide • hypertension • glomerulus • single nephron glomerular filtration rate
\end{abstract}

\section{Introduction}

The vascular endothelium is capable of synthesizing nitric oxide (NO) which acts on adjacent vascular smooth muscle cells to produce a cyclic GMP-dependent relaxation (1). NO is enzymatically synthesized from L-arginine, a process which can be antagonized by substituted L-arginine compounds such as $N$ monomethyl L-arginine (NMA) or $N$-nitro L-arginine methylester (NAME) which compete for the NO synthase (1). Studies in anesthetized rabbits, guinea pigs, and rats have demon-

This study was published in abstract form in J. Am. Soc. Nephrol. 2:471A, 1991.

Address correspondence to Dr. Christine Baylis, Department of Physiology, West Virginia University Health Sciences Center, Morgantown, WV 26506.

Received for publication 26 December 1991 and in revised form 25 February 1992.

1. Abbreviations used in this paper: BP, blood pressure; $K_{\mathrm{f}}$, glomerular capillary ultrafiltration coefficient; NAME, $N$-nitro L-arginine methylester; NMA, $N$-monomethyl L-arginine; NO, nitric oxide; $P_{\mathrm{GC}}$, glomerular blood pressure; $R_{\mathrm{A}}$, preglomerular arteriolar resistance; $R_{\mathrm{E}}$, efferent arteriolar resistance; SNGFR, single nephron GFR; $U_{p} V$, urinary protein excretion.

J. Clin. Invest.

(C) The American Society for Clinical Investigation, Inc.

$0021-9738 / 92 / 07 / 0278 / 04 \$ 2.00$

Volume 90, July 1992, 278-281 strated that acute blockade of NO synthesis leads to marked rises in systemic blood pressure (BP) (2-4), suggesting that tonic release of NO controls BP in the resting state. In the rat, acute blockade of NO synthesis produces a marked renal vasoconstriction with a major impact on glomerular hemodynamics, including substantial increases in glomerular capillary blood pressure $\left(P_{\mathrm{GC}}\right)(4,5)$. In the normal conscious chronically catheterized rat, studied in the basal state, acute NO blockade produces large increases in BP and renal vasoconstriction; effects that are acutely reversible with excess L-arginine $(6,7)$. The NO synthesis blocker NAME is water soluble and orally active, and when given in the drinking water to Brattleboro (hereditary diabetes insipidus) rats produces a prolonged increase in BP and renal vasoconstriction over many hours (8).

Based on these observations, we examined the possibility that long term blockade of NO synthesis, using the orally active drug NAME in the drinking water, might produce a model of systemic hypertension. Further, if the increase in $P_{\mathrm{GC}}$ seen with acute NO blockade $(4,5)$ is chronically maintained, then glomerular injury might evolve since glomerular capillary hypertension has been implicated in the pathogenesis of a variety of primary glomerular diseases (9). Accordingly, micropuncture studies were conducted in Munich Wistar rats chronically maintained on oral NAME for a 2-mo period and compared to age-matched controls.

\section{Methods}

Experiments were conducted on 14 male Munich Wistar rats purchased from Simonsen Laboratories, Gilroy, CA. At 4-6 mo of age, eight rats were placed on oral NAME (L-NAME, $5 \mathrm{mg} / 100 \mathrm{ml}$ drinking water, changed daily) for a continual 2-mo period. In preliminary studies, we determined that the presence of L-NAME in the drinking water did not influence drinking, and since normal rats drink $\sim 100 \mathrm{ml}$ $\mathrm{H}_{2} \mathrm{O} / \mathrm{kg}$ body weight (BW) per $24 \mathrm{~h}$, the dose of NAME was $\sim 5 \mathrm{mg} / \mathrm{kg}$ $\mathrm{BW}$ per $24 \mathrm{~h}$. Immediately before placing rats on oral NAME and at two weekly intervals thereafter, 24-h urine collections were made and urinary protein excretion measured by the Bradford method (10). A control group of six rats was aged over a similar time period, and terminal 24-h urine collections were made just before the acute experiment.

On the day of micropuncture, rats were anesthetized with intraperitoneal thiobarbiturate, Inactin $(120 \mathrm{mg} / \mathrm{kg})$, were placed on a temperature-controlled micropuncture table and temperature maintained at $37^{\circ} \mathrm{C}$ throughout the entire experiment. The rat was surgically prepared for glomerular micropuncture studies using the euvolemic (volume restored) preparation (11). Surgery included a tracheotomy, placement of intravenous lines for infusion of donor rat plasma (to maintain the euvolemic state), tritiated inulin $(100 \mu \mathrm{Ci} / \mathrm{h})$, and a femoral arterial line to monitor BP and collect blood samples. The left kidney was exposed through a ventral midline incision, the left ureter and left renal vein were catheterized, and the left kidney immobilized and prepared 
Table I. Summary of Whole Kidney and Glomerular Hemodynamics in Control Rats and Rats Subjected to Two Months of NO Blockade with Chronic Nitroarginine Methylester (NAME) Administration

\begin{tabular}{|c|c|c|c|c|c|c|c|c|c|c|c|c|c|c|c|}
\hline & $\mathrm{U}_{\mathrm{p}} \mathrm{V}$ & BP & GFR & RPF & FF & RVR & SNGFR & $Q_{\mathrm{A}}$ & $P_{\mathrm{GC}}$ & $P_{\mathrm{T}}$ & $C_{\mathrm{A}}$ & $C_{\mathrm{E}}$ & $R_{\mathrm{A}}$ & $R_{\mathrm{E}}$ & $K_{\mathrm{f}}$ \\
\hline & $m g / 24 h$ & $m m H g$ & \multicolumn{2}{|c|}{$\mathrm{ml} / \mathrm{min}$} & & $\begin{array}{c}m m H g / \\
m l \text { per min }\end{array}$ & \multicolumn{2}{|c|}{$n l / \min$} & \multicolumn{2}{|c|}{$m m H g$} & \multicolumn{2}{|c|}{$g \%$} & \multicolumn{2}{|c|}{$10^{10} \mathrm{dyn} \cdot \mathrm{s} \cdot \mathrm{cm}^{-5}$} & $\begin{array}{c}n l / s \text { per } \\
m m H g\end{array}$ \\
\hline Controls & 29 & 98 & 1.37 & 5.2 & 0.276 & 9.5 & 55.4 & 225 & 51 & 13 & 5.7 & 7.7 & 0.88 & 0.80 & $0.063^{\ddagger}$ \\
\hline$(n=6)$ & \pm 9 & \pm 2 & \pm 0.07 & \pm 0.6 & \pm 0.021 & \pm 1.0 & \pm 2.7 & \pm 26 & \pm 1 & \pm 1 & \pm 0.1 & \pm 0.2 & \pm 0.12 & \pm 0.12 & \pm 0.003 \\
\hline Chronic NAME & $79 *$ & $136^{*}$ & $0.90^{*}$ & $2.6^{*}$ & $0.379^{*}$ & $30.1^{*}$ & $33.8^{*}$ & $133^{*}$ & $60^{*}$ & 11 & 5.6 & 7.7 & $2.63^{*}$ & $1.88^{*}$ & $0.023^{*}$ \\
\hline$(n=8)$ & \pm 10 & \pm 4 & \pm 0.10 & \pm 0.5 & \pm 0.034 & \pm 5.6 & \pm 3.9 & \pm 20 & \pm 1 & \pm 1 & \pm 0.1 & \pm 0.3 & \pm 0.51 & \pm 0.32 & \pm 0.002 \\
\hline
\end{tabular}

All data given as mean \pm SE. ${ }^{*}$ Denotes significant difference from control by unpaired $t$ test. ${ }^{\ddagger}$ Mean minimum value since most control rats were at filtration pressure equilibrium.

for micropuncture as described previously (12). The surface of the kidney was illuminated and bathed in $0.9 \% \mathrm{NaCl}$ at $34-36^{\circ} \mathrm{C}$.

After equilibration, two exactly timed urine collections (25-30 min) were made, the volume measured, and midpoint blood samples taken from femoral artery and renal vein. Simultaneous micropuncture measurements were made in the superficial cortex as follows: exactly timed (2-3 $\mathrm{min})$ collection of fluid from five to six superficial proximal tubule segments; efferent arteriolar (postglomerular) blood collected by puncture of three to five superficial star vessels; hydrostatic pressures measured in surface glomerular capillaries, efferent arterioles, proximal tubules, and proximal segments of obstructed tubules (to measure $P_{\mathrm{GC}}$ using the indirect stop flow pressure method, described previously [13]). The activity of tritiated inulin was measured in aliquots of arterial and renal venous plasma, urine, and the entire tubule fluid sample, and allowed calculation of single nephron GFR (SNGFR), GFR, and renal plasma flow (RPF). Protein concentration of systemic and efferent arteriolar plasma was measured using a microadaptation of the Lowry method (14).

All of these measurements permit calculations of preglomerular and efferent arteriolar oncotic pressures, preglomerular and efferent arteriolar resistances $\left(R_{\mathrm{A}}\right.$ and $\left.R_{\mathrm{E}}\right)$, and the glomerular capillary ultrafiltration coefficient $\left(K_{\mathrm{f}}\right)$. These techniques have been described in detail by us elsewhere $(12,13)$. At the end of the functional experiment, the rat was killed, the left kidney removed, weighed, bisected longitudinally, and fixed in $10 \%$ buffered formalin. Later, the kidney was dehydrated in alcohol, blocked in paraffin wax, and 5- $\mu \mathrm{m}$ thick sections were cut and stained with periodic acid Schiff and hemotoxylin and eosin counterstain. Sections were examined on a blinded basis for the level of glomerular sclerosis (using the 0-4+ injury scale [15]) and microvascular injury.

The statistical comparison of functional data was by unpaired $t$ test, except for the serial comparisons of urinary protein excretion $\left(U_{p} V\right)$ which was by analysis of variance (ANOVA), and the morphologic data was analyzed using the Wilcoxon two sample test. Statistical significance is assumed where $P<0.05$.

\section{Results}

The proteinuria evolved slowly in chronically NO-blocked rats; 24 -h urinary protein excretion $\left(\mathrm{U}_{\mathrm{p}} \mathrm{V}\right)$ was $36 \pm 3 \mathrm{mg}$ in the basal state, before NO blockade, and $49 \pm 5,73 \pm 9$, and $69 \pm 9$ mg at weeks 2, 4, and 6 of NO blockade $(P<0.05$ basal vs weeks 4 and 6). As shown in Table $I$ at week $8, U_{p} V$ was significantly increased in chronically NO-blocked rats vs agematched controls (and vs basal in the NO-blocked group, $P$ $<0.05$ ). Mean body weight and left kidney weight was $387 \pm 14$ $\mathrm{g}$ and $1.3 \pm 0.1 \mathrm{~g}$ in controls, and values were similar in chronically NO-blocked rats, $358 \pm 6 \mathrm{~g}$ and $1.3 \pm 0.1 \mathrm{~g}$, respectively.

Mean BP was elevated vs untreated control rats (Table I), despite the fact that these observations were made under barbiturate-induced general anesthesia. Whole kidney and glomerular hemodynamics data are summarized in Table I. The GFR was reduced in chronically NO-blocked vs control animals, and RPF was reduced by a disproportionately greater amount such that filtration fraction (FF) was elevated. The renal vascular resistance $(\mathrm{RVR})$ was $\sim 3 \mathrm{x}$ the control value in chronically NO-blocked rats (Table I). Essentially similar findings were observed at the single nephron level; SNGFR and glomerular plasma flow $\left(Q_{\mathrm{A}}\right)$ were significantly reduced in chronically NOblocked vs control rats. The $P_{\mathrm{GC}}$ was significantly higher in chronically NO-blocked animals, while pressure in the proximal tubule (which is similar to that in Bowman's space) was not different between the two groups. The plasma protein concentration $\left(C_{\mathrm{A}}\right)$ and efferent arteriolar protein concentration $\left(C_{\mathrm{E}}\right)$ were also similar between groups; thus, values of pre- and postglomerular oncotic pressures (not shown) were also similar. Values for segmental arteriolar resistances were greatly elevated in the chronically NO-blocked rats, and both $R_{\mathrm{A}}$ and $R_{\mathrm{E}}$ were greater than in control. Despite the marked rise in $R_{\mathrm{A}}, P_{\mathrm{GC}}$ increased both because of the increase in BP and in $R_{\mathrm{E}}$. The $K_{\mathrm{f}}$ was significantly reduced in chronically NO-blocked rats vs controls.

Morphologic evaluation revealed that there was significantly greater glomerular sclerotic injury in chronically NOblocked rats vs controls. The severity of glomerular injury, using the $0-4+$ scale is shown in Table II, and it is clear that the chronically NO-blocked rats exhibit greater injury vs controls. Based on the data in Table II, an overall glomerulosclerosis injury score can be calculated as described by Raij et al. (15), and the chronically NO-blocked rats show significantly greater

Table II. Semiquantitative Histologic Injury Score (0 to 4+) of controls and Chronically NO-blocked rats

\begin{tabular}{lccccc}
\hline & \multicolumn{5}{c}{ Injury score } \\
\cline { 2 - 6 } \multicolumn{1}{c}{ Group } & 0 & $1+$ & $2+$ & $3+$ & $4+$ \\
\hline Control & $93.5 \pm 1.1$ & $4.2 \pm 0.8$ & $1.2 \pm 0.5$ & $1.0 \pm 0.4$ & $1.0 \pm 0.3$ \\
NO blocked & $80.6 \pm 1.9$ & $12.4 \pm 0.9$ & $3.6 \pm 0.5$ & $1.0 \pm 0.3$ & $2.8 \pm 0.6$ \\
$P<$ & 0.001 & 0.001 & 0.05 & NS & 0.05 \\
& & & & &
\end{tabular}

Injury scale: $0=$ no injury; $1+=$ up to $25 \%$ of the glomerulus involved; $2+=\sim 50 \%$ involvement; $3+=\sim 75 \%$ involvement; $4+=$ $\sim 100 \%$ involvement. 
overall damage with a score $=34 \pm 4$ vs controls $=12 \pm 2 ; P$ $<0.01$. Mild to moderate medial expansion of arterioles in the kidney cortex were present without significant periarteriole fibrosis.

\section{Discussion}

In these experiments we have demonstrated that chronic NO blockade produces sustained systemic hypertension, confirming the major role of NO in maintenance of normal vascular tone (1-4). There have been several other recent preliminary reports of persistent increases in blood pressure with chronic NO inhibition. Ribeiro et al., using $\sim 10$-fold higher dose than we employed in the rat, reported a significantly greater hypertension (about $170 \mathrm{mmHg}$ mean) after 4-6 wk of continual NO blockade with NAME (16). We have recently obtained preliminary data suggesting that there is a dose dependency to the magnitude of the systemic hypertension achieved with chronic NO blockade (unpublished observations). Chander et al. used a different NO synthesis inhibitor ( $N$-nitro L-arginine) in the stroke-prone spontaneously hypertensive rat. These animals are normally extremely hypertensive (systolic blood pressures about $230 \mathrm{mmHg}$ ), and not surprisingly chronic NO blockade for $10 \mathrm{~d}$ did not significantly worsen the hypertension; however, the incidence of vascular lesions in this hypertensive rat model was greatly increased by chronic NO blockade (see below) (17). Two groups have reported production of a dog model of chronic NO blockade. Salazar et al. (18) administered a low dose of continual intravenous NAME, $\sim 0.15 \mathrm{mg} / \mathrm{kg}$ per d, to dogs for $5 \mathrm{~d}$ continually. In dogs on normal sodium intake, this had no effect on mean BP; however, in dogs maintained on a high sodium intake, BP increased significantly (by $13 \mathrm{mmHg}$ ). Manning et al., using a much larger dose of another NO synthesis inhibitor, nitro L-arginine (about $15 \mathrm{mg} / \mathrm{kg}$ per $24 \mathrm{~h}$ ), showed that a $16-\mathrm{mmHg}$ increase in mean BP was maintained over an 8-d period, an effect that was reversible with excess L-arginine, denoting specificity of the blocker (19). The limited amount of preliminary data currently available suggests that the maximum increase in BP to be elicited with NO blockade in the dog is less than that in the rat, but these results await further confirmation. The data from the present and other preliminary studies (16-19) certainly suggests that chronic inhibition of NO synthesis produces increases in BP. Furthermore, it was recently shown that chronic supplementation with the NO precursors L-arginine or L-citrulline prevents the development of hypertension in genetically salt-sensitive rats fed a high salt diet (20). Whether chronic NO deficiency contributes to systemic hypertension in man is not yet clear, although this is possible, particularly in low renin, salt-dependent hypertension.

In addition to systemic hypertension we observed increased glomerular injury in the presence of chronic NO blockade, with an increased incidence of focal glomerular sclerosis after 2 mo of moderate systemic hypertension with sustained glomerular capillary hypertension. In the study by Ribero et al., segmental fibrinoid necrosis was reported in glomeruli of chronically NO-blocked rats; also arteriolar hypertrophy and in severely hypertensive rats, focal arteriolar obliteration was seen (16). In these rats, glomerular blood pressure was not measured but renal vasoconstriction and increased filtration fraction was reported, which is suggestive of glomerular hypertension. In the chronically NO-blocked stroke-prone SHR, there was a large increase in $U_{p} V$ and greater incidence of vascular lesions and glomerular injury, compared with control stroke-prone SHRs (17). Unfortunately, in this model no renal functional measurements were available.

The mechanism causing glomerular injury during chronic NO blockade may be glomerular capillary hypertension. Glomerular hypertension has been causally implicated in the pathogenesis of glomerular sclerotic injury in a variety of glomerular diseases (9), in particular, ablation of renal mass and in an experimentally induced model of type I diabetes. In both of these situations, $P_{\mathrm{GC}}$ increases quite early in the course of the disease, and maneuvers that lower $P_{\mathrm{GC}}$ also protect the glomerulus from injury (9). In the present model, $P_{\mathrm{GC}}$ may be assumed to increase immediately, since we and others have shown that acute systemic NO blockade leads to systemic and glomerular hypertension within minutes of administration of the NO synthesis inhibitors $(4,5)$. An alternative or additional mechanism that might be responsible for producing glomerular injury as a result of chronic NO blockade may relate to the antimitogenic action of NO on the glomerular mesangial cell (21). Mesangial expansion precedes the development of glomerular sclerotic injury, and a causal link in this process has been suggested (22). Thus, chronic NO inhibition may provide a permissive mesangial hypertrophic stimulus. This could explain the worsening of vascular and glomerular damage in the stroke-prone SHR (17), where no worsening of the hypertension occurred with chronic NO blockade.

In conclusion, these present studies describe a novel model of systemic hypertension with glomerular capillary hypertension and glomerular injury due to chronic NO blockade. Further study of this model should yield significant insights into the mechanisms of development of systemic hypertension and glomerular damage.

\section{Acknowledgments}

The excellent technical assistance of Lennie Samsell and secretarial/ editorial assistance of Lisa Bradley are gratefully acknowledged.

These studies were supported by National Institutes of Health grant 31933.

\section{References}

1. Moncada, S., R. M. J. Palmer, and E. A. Higgs. 1991. Biosynthesis and endogenous roles of nitric oxide. Pharmacol. Rev. 43:109-142.

2. Rees, D. D., R. M. J. Palmer, and S. Moncada. 1989. Role of endotheliumderived nitric oxide in the regulation of blood pressure. Proc. Natl. Acad. Sci. USA. 86:3375-3378.

3. Aisaka, K., S. S. Gross, O. W. Griffith, and R. Levi. 1989. $N^{G}$-Methylarginine, an inhibitor of endothelium-derived nitric oxide synthesis, is a potent pressor agent in the guinea pig: does nitric oxide regulate blood pressure in vivo? Biochem. Biophys. Res. Commun. 160:881-885.

4. Zatz, R., and G. de Nucci. 1991. Effects of acute nitric oxide inhibition of rat glomerular microcirculation. Am. J. Physiol. 30:F360-F363.

5. Deng, A., L. Samsell, and C. Baylis. 1991. Locally produced endothelialderived relaxing factor (EDRF) controls preglomerular $\left(R_{A}\right)$ arteriolar tone and the glomerular capillary ultrafiltration coefficient, $K_{\mathrm{f}}$. J. Am. Soc. Nephrol. 2:503A.

6. Gardiner, S. M., A. M. Compton, T. Bennet, R. M. J. Palmer, and S. Moncada. 1990. Control of regional blood flow by endothelium-derived nitric oxide. Hypertension (NY). 15:486-492.

7. Baylis, C., P. Harton, and K. Engels. 1990. Endothelial derived relaxing factor controls renal hemodynamics in the normal rat kidney. J. Am. Soc. Nephrol. 1:875-881.

8. Gardiner, S. M., A. M. Compton, T. Bennett, R. M. J. Palmer, and S. Moncada. 1990. Regional haemodynamic changes during oral ingestion of $N^{2}$. monomethyl-L-arginine or $N^{3}$-nitro-L-arginine methyl ester in conscious Brattleboro rats. Br. J. Pharmacol. 101:10-12. 
9. Brenner, B. M. 1985. Nephron adaptation to renal injury or ablation. Am. J. Physiol. 249:F324-F337.

10. Bradford, M. M. 1976. A rapid and sensitive method for the quantitation of microgram quantities of protein utilizing the principle of protein-dye binding. Anal. Biochem. 72:248-254.

11. Ichikawa, I., D. A. Maddox, M. C. Cogan, and B. M. Brenner. 1978. Dynamics of glomerular ultrafiltration in euvolemic Munich Wistar rats. Renal Physiol. 1:121-131.

12. Baylis, C., W. M. Deen, B. D. Myers, and B. M. Brenner. 1976. Effects of some vasodilator drugs on transcapillary fluid exchange in renal cortex. $\mathrm{Am}$. J. Physiol. 230:1148-1158.

13. Baylis, C. 1989. Immediate and long-term effects of pregnancy on glomerular function in the SHR. Am. J. Physiol. 257:F1140-F1145.

14. Lowry, O. H., N. J. Rosebrough, A. L. Farr, and R. J. Randall. 1951. Protein measurement with the Folin phenol reagent. J. Biol. Chem. 193:265-275.

15. Raij, L., S. Azar, and W. Keane. 1984. Mesangial immune injury, hypertension, and progressive glomerular damage in Dahl rats. Kidney Int. 26:137143.
16. Ribeiro, M. O., G. de Nucci, and R. Zatz. 1991. Persistent arterial hypertension by chronic blockade of nitric oxide synthesis. J. Am. Soc. Nephrol. 2:512A.

17. Chander, P. N., P. O'Brien, and C. T. Stier. 1991. N-nitro-L-arginine (L-NMA) markedly accelerates the development of malignant nephrosclerosis in stroke-prone SHR (SHRSP). J. Am. Soc. Nephrol. 2:501A.

18. Salazar, F. J., J. M. Pinilla, A. Alberola, J. C. Romero, and T. Quesada. 1991. Salt-induced increase in blood pressure during chronic inhibition of EDRF synthesis. Hypertension (NY). 18:387A.

19. Manning, R. D., L. Hu, H. L. Mizelle, J. P. Montani, and M. W. Norton. 1991. Effects of long-term blockade of endothelial-derived relaxing factor (EDRF) on the cardiovascular system. Hypertension (NY). 18:391 A.

20. Chen, P. Y., and P. W. Sanders. 1991. L-arginine abrogates salt-sensitive hypertension in Dahl/Rapp rats. J. Clin. Invest. 88:1559-1567.

21. Garg, U. C., and A. Hassid. 1989. Inhibition of rat mesangial cell mitogenesis by nitric oxide-generating vasodilators. Am. J. Physiol. 257:F60-F66.

22. Mauer, S. M., M. W. Steffes, E. N. Ellis, D. E. R. Sutherland, D. M Brown, and F. C. Goetz. 1984. Structural-functional relationships in diabetic nephropathy. J. Clin. Invest. 74:1143-1155. 